\title{
Cardiac Troponin T: An Early Molecule Marker of Normalization of Left Ventricular Ejection Fraction in Patients with Peripartum Cardiomyopathy
}

\author{
Yan-Bo Li ${ }^{a}$ Cheng-Lin Hu ${ }^{b}$ Jia-Ming Zhang ${ }^{c}$ Yong-Guang Zou ${ }^{d}$ \\ Yan-Hong Tang ${ }^{\mathrm{b}}$ Jiang-Bing Chen ${ }^{\mathrm{b}}$ Qi-Zhu Tang ${ }^{\mathrm{b}}$ Cong-Xin Huang ${ }^{\mathrm{b}}$ \\ Departments of a Obstetrics and Gynecology and ${ }^{b}$ Cardiology, Renmin Hospital of Wuhan University, \\ 'Department of Cardiology, Wuhan Xiehe Hospital, Huazhong University of Science and Technology, Wuhan, and \\ ${ }^{\mathrm{d}}$ Department of Cardiology, Xiaogan General Hospital, Xiaogan, PR China
}

\section{Key Words}

Peripartum cardiomyopathy · Troponin T • Ventricular function

\begin{abstract}
Objectives: It was the aim of this study to assess the prognostic role of the initial concentration of cardiac troponin T (CTnT) for normalization of left ventricular ejection fraction (LVEF) in peripartum cardiomyopathy (PPCM). Methods: The study of 111 patients with newly diagnosed PPCM was a prospective observational clinical trial. Clinical assessment and echocardiography were done during the first 2 weeks as well as 6 months after the onset of PPCM. The initial concentration of CTnT was measured during the first 2 weeks after onset of PPCM. Results: Analysis by receiver operating characteristic curve indicated that initial cTnT concentrations may provide prognostic information superior to left ventricular end-diastolic anterior-posterior diameter or LVEF. The best cutoff value of initial cTnT $\leq 0.02 \mathrm{ng} / \mathrm{ml}$ predicted normalization of LVEF with a sensitivity of $81.0 \%$ and a specificity of $67.9 \%$. Multivariate logistic regression analysis identified initial cTnT as the only independent predictor of 6-month normalization of LVEF. Conclusions: The initial cTnT concentration is a potential independent predictor of normalization of LVEF within 6 months of onset of PPCM and may provide prognostic information superior to left ventricular end-diastolic anterior-posterior diameter and LVEF.

Copyright $\odot 2007$ S. Karger AG, Basel
\end{abstract}

\section{Introduction}

Peripartum cardiomyopathy (PPCM) is a rare cardiomyopathy of unknown cause that occurs in pregnant females, most commonly in the early postpartum period. It is similar to dilated cardiomyopathy (DCM) in terms of symptoms, histopathology and treatment, but is characterized by onset at a younger age and a better outcome with a high rate of spontaneous normalization of left-ventricular size and function within 6 months of the onset of the disease [1-5].

The long-term prognosis of PPCM appears to be related to the rapid recovery of ventricular function [5-7]. Many of the patients whose ventricular function returned to normal have resumed active lives, whereas patients who maintain persistent ventricular dysfunction for 6 months or longer have an extremely poor prognosis. Prompt recognition of the condition at diagnosis is required for initiation of appropriate medical management and counseling regarding future pregnancies. Thus, early risk stratification and prognostication are crucial. However, only limited data are available [8]. Although being an important diagnostic tool in PPCM, the prognostic value of echocardiography in PPCM seems to be poor [9].

The serum cardiac troponin $\mathrm{T}(\mathrm{c} \operatorname{Tn} \mathrm{T})$, a specific and highly sensitive marker of myocardial injury, is a suitable candidate marker molecule to monitor congestive heart

Cong-Xin Huang, MD

Department of Cardiology, Renmin Hospital of Wuhan University

238 Jiefang Road

Wuhan 430060 (PR China)

Tel. +86 278804 1911, Fax +86 278823 7987, E-Mail yanboli72@yahoo.com.cn 
failure from a structural perspective [10]. Its prognostic values have been reported in acute coronary syndromes $[11,12]$, idiopathic DCM $[13,14]$, hypertrophic cardiomyopathy [15] and acute myocarditis [16]. However, its prognostic value in PPCM is still unknown. The present study was designed to assess its prognostic role and provide cTnT cutoff values by use of receiver operating characteristic (ROC) curves for normalization of left ventricular ejection fraction (LVEF) in PPCM.

\section{Methods}

\section{Study Population}

This clinical trial was designed as a prospective observational study. The criteria for the diagnosis of PPCM included the development of congestive heart failure during the last month of pregnancy or the first 5 months postpartum, the absence of another identifiable cause of heart failure, and evidence of depressed left ventricular function, defined as an $\mathrm{LVEF}<40 \%$, as measured by echocardiography $[6,17]$. Patients who visited our hospitals during the first 2 weeks after they had experienced their first episode of heart failure and survived over 6 months after onset of PPCM were eligible if they could give written informed consent. According to the 6-month outcome, patients were divided into two groups: normalization of LVEF (group 1) and persistent ventricular dysfunction (group 2).

The clinical status was determined by the New York Heart Association function classification. cTnT was analyzed with the third-generation TnT test (Troponin T STAT). The third-generation TnT test uses the same monoclonal antibodies (M11.7 and M7) as the second-generation test but is standardized with human recombinant cTnT instead of bovine cTnT (Roche Diagnostics) [18]. The lower detection limit of this assay is $0.01 \mu \mathrm{g} / \mathrm{l}$. LVEF and left ventricular end-diastolic anterior-posterior diameter (LVDd) were measured echocardiographically by 2 experts unaware of this study protocol and whose measurements were averaged.

\section{Definitions and Study End Points}

The primary study end point was normalization of LVEF during 6 months. The normalizations of LVEF and LVDd were defined as LVEF $>50 \%$ [11] and LVDd $\leq 48 \mathrm{~mm}$ [12].

\section{Study Protocol}

Clinical assessment and echocardiography were done during the first 2 weeks as well as 6 months after the onset of PPCM. The initial concentration of $\mathrm{cTnT}$ was measured during the first 2 weeks after the onset of PPCM. Pharmacological treatment includes angiotensin-converting enzyme inhibitors, angiotensin receptor blockers and $\beta$-adrenergic blockers, diuretics, digoxin and anticoagulation. Angiotensin-converting enzyme inhibitors and angiotensin receptor blockers should be avoided during pregnancy because of severe adverse neonatal effects. This trial was done at three teaching hospitals in central China, i.e. Renmin Hospital of Wuhan University, Wuhan Xiehe Hospital and Xiaogan General Hospital. The local research and ethics committees approved this protocol. Written informed consent was obtained from all patients.

\section{Statistical Analysis}

Results are presented as means \pm SD for continuous normally distributed variables, as medians (lower, upper quartile) for continuous non-normally distributed data, and as percentages for categorical data. We tested continuous variables by one-way ANOVA for between-group comparisons (with the baseline value as the covariable) and Student's t test for within-group comparisons. If a normal distribution of variables was not present, the Mann-Whitney test was used for between-group differences and the Wilcoxon signed ranks test for within-group differences. Group comparison for categorical variables was performed by the $\chi^{2}$ test with continuity correction or Fisher's exact test as appropriate. The data were analyzed using SPSS for windows 11.0 (SPSS Inc.). Multivariate logistic regression was performed on univariately significant variables. The ability of these significant variables to predict outcome was assessed by ROC curve analysis. Sensitivity was plotted against 1 - specificity to give the ROC plot. The point of inflection of the ROC curve allows selection of the best tradeoff between sensitivity and specificity. The nearer the curve to the top left corner of the graph, the better the test. ROC was done using MedCalc 7.5 (MedCalc software). All statistical tests were two-sided. A p value $<0.05$ was considered significant.

\section{Results}

A total of 111 consecutive patients with newly diagnosed PPCM were recruited between 1 January 2003 and 1 May 2005. The mean age at diagnosis was $28.1 \pm 6.2$ years. All women were initially seen for pulmonary edema. Nineteen women (17\%) were first seen antepartum and $92(83 \%)$ postpartum. None of the patients had a previous history of heart disease. LVEF at the time of diagnosis was $26.62 \pm 5.90 \%$ and improved to $45.24 \pm 13.85 \%$ $(\mathrm{p}<0.0001)$ at follow-up. Fifty-eight of 111 patients $(52 \%)$ had a return of LVEF to normal (group 1) and 53/111 patients (48\%) maintained left ventricular dysfunction (group 2). Blood samples for cTnT measurements were taken $8.3 \pm 3.0$ days (group 1) and $7.3 \pm 2.2$ days (group 2) after the onset of PPCM ( $p=0.718)$.

At baseline, there was no difference between the two groups with respect to demographic and clinical parameters (i.e. age, body mass index, parity, incidence of twins, heart rate, systolic and diastolic blood pressure, antepartum or postpartum, clinical presentation, New York Heart Association functional class, nutritional status, mode of initial therapy, and blood creatine; data not shown). However, there were different median (lower, upper quartile) cTnT concentrations [0.01 $(0.01,0.02)$ vs. $0.05(0.02,0.06) \mathrm{ng} / \mathrm{ml} ; \mathrm{p}<0.0001], \operatorname{LVDd}(56.05 \pm 3.62$ vs. $58.94 \pm 6.32 \mathrm{~mm} ; \mathrm{p}=0.003)$ and $\operatorname{LVEF}(27.71 \pm 5.66$ vs. $25.43 \pm 5.98 \% ; \mathrm{p}=0.042)$ in groups 1 and 2 . 
Table 1. Effect on sensitivity and specificity of increasing the cutoff value for $\mathrm{c} T n \mathrm{~T}$

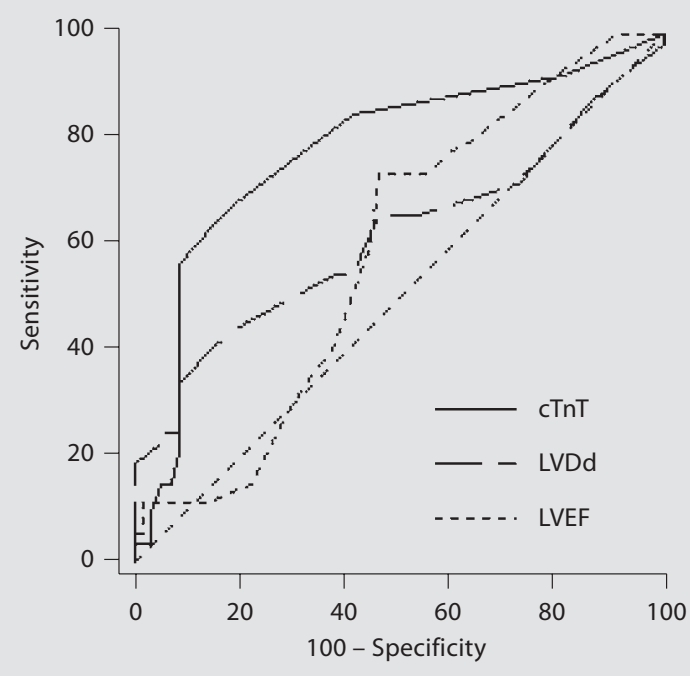

Fig. 1. ROC plots of initial cTnT, LVDd and LVEF to predict normalization of LVEF after follow-up.

To predict normalization of LVEF, ROC was done. The area under the ROC curve for $\mathrm{c} \operatorname{TnT}(0.779$, range 0.690 $0.852)$, LVDd (0.617, range $0.520-0.708)$ or LVEF $(0.583$, range $0.485-0.676$ ) is shown in figure 1 (cTnT vs. LVDd,
cTnT vs. LVEF, and LVDd vs. LVEF, $\mathrm{p}=0.013,0.004$ and 0.631 , respectively), indicating that cTnT concentrations have a greater predictive capacity for normalization of LVEF than LVDd or LVEF.

The third-generation cTnT assay has both an improved imprecision profile and a lower detection limit. Therefore, a detailed evaluation of the ROC curve was performed. The clinical sensitivity for cTnT was examined using cutoff values (table 1). Increase in the cutoff value resulted in improvement in sensitivity but loss of specificity, with an optimal tradeoff of $\leq 0.02 \mathrm{ng} / \mathrm{ml}$ for predicting LVEF normalization.

Using multivariate logistic regression analysis, initial cTnT $\leq 0.02 \mathrm{ng} / \mathrm{ml}$ (OR 9.05; 95\% CI 3.78-21.68; p < $0.0001)$ was the only independent predictor of normalization of LVEF 6 months after onset of PPCM.

In 111 recruited patients, there were 64 patients with cTnT concentrations $\leq 0.02 \mathrm{ng} / \mathrm{ml}$ and 47 patients with cTnT concentrations $>0.02 \mathrm{ng} / \mathrm{ml}$. At follow-up, more normalized LVDd (51.6 vs. 23.4\%; OR 3.49; $95 \%$ CI 1.518.03; $\mathrm{p}=0.003$ ) or LVEF (73.4 vs. $23.4 \%$; OR 9.05; 95\% CI 3.78-21.68; $\mathrm{p}<0.0001)$ occurred in patients with cTnT $\leq 0.02 \mathrm{ng} / \mathrm{ml}$ than in patients with $\mathrm{cTnT}>0.02 \mathrm{ng} / \mathrm{ml}$. 


\section{Discussion}

To our knowledge, this is the first study to compare cTnT, LVDd and LVEF in the prognosis of PPCM. In this 6-month course study, we sought early predictors of prognosis in patients with recently diagnosed PPCM. The most significant finding is that cTnT concentration measured during the first 2 weeks after the onset of PPCM is a potential independent predictor of normalization of LVEF within 6 months of onset of PPCM and may provide prognostic information superior to LVDd and LVEF.

The clinical presentation of patients with PPCM is similar to that of patients with systolic congestive heart failure, but the outcome of patients with PPCM is highly variable. There appears to be an initial high-risk period with $25-50 \%$ of women dying within the first 3 months postpartum. Approximately $1 / 3$ to $1 / 2$ of the patients have a return to normal heart size and function within 6-12 months after the onset of their disease [5, 19-22]. Many of the patients whose heart size returned to normal have resumed active lives. Patients who maintain cardiomegaly for 6 months or longer have an extremely poor prognosis. These patients continue to have an abnormal electrocardiogram and gallop rhythms, and most of them have recurrent episodes of congestive heart failure leading to death within a few years. In our study, despite treatment with angiotensin-converting enzyme inhibitors and $\beta$-blockers, only $52 \%$ of the patients had a return of LVEF to normal, and the other patients maintained left ventricular dysfunction within 6 months of onset of PPCM.

The prognostic value of echocardiography in PPCM seems to be poor or uncertain. A previous study [23] revealed that patients with severe myocardial dysfunction due to PPCM are unlikely to regain normal cardiac function on follow-up, and a fractional shortening value $<20 \%$ and an LVDd $\geq 6 \mathrm{~cm}$ at the time of diagnosis are associated with a more than 3-fold higher risk of persistent left ventricular dysfunction [24]. The study by Dorbala et al. [8] revealed that inotropic contractile reserve during dobutamine stress echocardiography accurately correlates with subsequent recovery of left ventricular function and confers a benign prognosis, whereas the baseline LVEF did not correlate with follow-up LVEF. Another study [25] revealed that the initial severity of the left ventricular systolic dysfunction or dilatation is not necessarily predictive of the long-term functional outcome. Left ventricular echocardiographic features at diagnosis were unable to individually predict who would eventually recov- er, although a statistically significant difference occurred at diagnosis between the recovered and nonrecovered group for mean ejection fraction (28 vs. $23 \%$ ) and fractional shortening (17 vs. 14\%) [9]. In our study, univariate analysis revealed that initial LVDd and LVEF are significant predictors of short-term outcome, but multivariate analysis could not reveal that they could independently predict normalization of left ventricle function. The area under the ROC curve was relatively small $(<0.70)$, indicating that their predictive capacity is poor.

The measurement of serum concentrations of cardiac cTnT is a simple, useful method to detect myocyte injury [13]. Myocardial biopsies in PPCM revealed that the most prominent findings were hypertrophy of myocardial fibers and varying degrees of fibrosis. There appeared to be no significant histological differences between the patients with normal heart size and those with persistent cardiomegaly after the onset of heart failure [5]. In our study, the initial cTnT concentration was lower in patients with a return to normal heart function than in patients who maintained cardiomegaly within 6 months. Moreover, at follow-up, the normalization rate of LVDd or LVEF was higher in patients with cTnT concentrations $\leq 0.02 \mathrm{ng} / \mathrm{ml}$. Serum cTnT in PPCM seems to indicate ongoing myocyte degeneration. This can partly explain the difference in the two groups with respect to hemodynamic change, left ventricular remodeling and different prognosis. The mechanisms of myocyte injury in PPCM are not fully understood. It may be caused by virus infection, autoimmune mechanisms, hormonal changes, genetic disorders and toxemia [5]. A possible correlation between these factors and cTnT concentrations requires further study. Excessive alcoholic intake has not been important. Although some authors have mentioned poor nutrition [26], patients reported by others have been well nourished [5, 19]. Renal failure is also a recognized cause of raised cTnT concentration [27], but the results were not altered by exclusion of patients with a serum creatine concentration $>0.2 \mathrm{mmol} / \mathrm{l}$.

In our still unpublished study, univariate analysis revealed that serum cTnT concentration measured during the first 2 weeks after the onset of PPCM is correlated negatively with follow-up LVEF. In the present study, multivariate logistic regression analysis identified that $c \operatorname{TnT} \leq 0.02 \mathrm{ng} / \mathrm{ml}$ is the only independent predictor of 6-month normalization of LVEF. The area under the curve analyzed by ROC for cTnT was bigger than that for LVDd or LVEF, indicating that cTnT concentrations may provide prognostic information superior to LVDd or LVEF. A single initial cTnT measurement can be used to 
estimate myocardial cell injury that impacts LVEF normalization.

Long-term prognosis appears to be related to left ventricular chamber dimension and function at diagnosis and recovery $[5,28]$. About half the patients of PPCM recover without any complications, and their medical therapy can be tapered off or discontinued over a period of 6-12 months. Persistence of disease after 6 months indicates irreversible cardiomyopathy and portends worse chances of survival $[29,30]$. It can be presumed that cTnT might also serve as a useful new prognostic indicator for long-term outcomes in PPCM. This seemed to be important to PPCM. Clinicians must be aware of this problem in order to provide prompt diagnosis and effective treatment that will ensure a favorable return of normal heart size and normal left ventricular function.

The prognostic values of cTnT in other kinds of heart failure have also been studied in various clinical settings [11, 13-16, 31]. Abnormal cTnT concentrations were detected in $>50 \%$ of outpatients with advanced congestive heart failure. Conversely, the presence of troponin in decompensated congestive heart failure was also associated with more profound clinical and hemodynamic deterioration, more severe left ventricular remodeling and worse short- and long-term prognosis [31]. In patients with DCM and secondary cardiomyopathy, there was a subgroup of patients with raised concentrations of serum cTnT, for whom short-term prognosis was poor [14]. Persistently increased TnT concentrations in DCM suggest ongoing subclinical myocyte degeneration associated with deterioration of the patients' clinical status [13]. An increase in TnT serum concentrations in hypertrophic cardiomyopathy points to subclinical myocyte injury. During an average of 21.5 months of follow-up, patients with an increased serum cTnT level had a decrease in ventricular fractional shortening and ventricular septal thickness on echocardiogram [15]. In acute myocarditis, the cTnT level is sig- nificantly higher than that in DCM. However, it had no significant effect on outcomes of the patients [16].

Some other molecular markers may also have predictive values in PPCM. Cytokine and sFas levels are elevated in patients with PPCM [32]. Baseline levels of C-reactive protein correlate positively with baseline LVDd and left ventricular end-systolic diameter and inversely with LVEF. Fas/Apo-1 can predict mortality [21]. The outcome of the predictive capacity of these molecular markers needs further study.

A limitation of this work is the relatively small sample size of the population and short-term course. Our sample was relatively small, especially for the comparison of two ROC curves. This could have affected our results. Despite the limited number of patients, we detected significant differences in prognostic values between cTnT and LVDd or LVEF. A large study of the relation between serum concentrations of cTnT and long-term outcomes is wanted. In the meantime, our conclusions are valid only for the subpopulation of patients with newly diagnosed PPCM. Moreover, this study was not designed to examine the effects of drug treatment on the evolution of serum cTnT concentrations. Larger clinical drug trials including the monitoring of $\mathrm{cTnT}$ concentrations should be planned.

\section{Conclusions}

cTnT concentration measured during the first 2 weeks after the onset of PPCM is an early molecule marker and the only potential independent predictor of normalization of LVEF within 6 months of onset of PPCM and may provide prognostic information superior to LVDd and LVEF. The best cutoff value of cTnT $\leq 0.02 \mathrm{ng} / \mathrm{ml}$ can predict normalization of LVEF with a sensitivity of $81.0 \%$ and a specificity of $67.9 \%$.

\section{References}

1 Phillips SD, Warnes CA: Peripartum cardiomyopathy: current therapeutic perspectives. Curr Treat Options Cardiovasc Med 2004;6: 481-488.

-2 Murali S, Baldisseri MR: Peripartum cardiomyopathy. Crit Care Med 2005;33:S340S346.
-3 de Souza JL Jr, de Carvalho Frimm C, Nastari L, Mady C: Left ventricular function after a new pregnancy in patients with peripartum cardiomyopathy. J Card Fail 2001;7: 30-35.

$\checkmark 4$ Veille JC: Peripartum cardiomyopathies: a review. Am J Obstet Gynecol 1984;148:805818.

5 Demakis JG, Rahimtoola SH, Sutton GC, Meadows WR, Szanto PB, Tobin JR, Gunnar RM: Natural course of peripartum cardiomyopathy. Circulation 1971;44:1053-1061.
6 Lampert MB, Lang RM: Peripartum cardiomyopathy. Am Heart J 1995;130:860-870.

7 Pearson GD, Veille JC, Rahimtoola S, Hsia J, Oakley CM, Hosenpud JD, Ansari A, Baughman KL: Peripartum cardiomyopathy: National Heart, Lung, and Blood Institute and Office of Rare Diseases (National Institutes of Health) workshop recommendations and review. JAMA 2000;283:1183-1188. 
$>8$ Dorbala S, Brozena S, Zeb S, Galatro K, Homel P, Ren JF, Chaudhry FA: Risk stratification of women with peripartum cardiomyopathy at initial presentation: a dobutamine stress echocardiography study. J Am Soc Echocardiogr 2005;18:45-48.

-9 Fett JD, Christie LG, Carraway RD, Murphy JG: Five-year prospective study of the incidence and prognosis of peripartum cardiomyopathy at a single institution. Mayo Clin Proc 2005;80:1602-1606.

-10 Missov E, Mair J: A novel biochemical approach to congestive heart failure: cardiac troponin T. Am Heart J 1999;138:95-99.

- 11 Elkayam U, Akhter MW, Singh H, Khan S, Bitar F, Hameed A, Shotan A: Pregnancy-associated cardiomyopathy: clinical characteristics and a comparison between early and late presentation. Circulation 2005;111: 2050-2055.

-12 Chapa JB, Heiberger HB, Weinert L, Decara J, Lang RM, Hibbard JU: Prognostic value of echocardiography in peripartum cardiomyopathy. Obstet Gynecol 2005;105:13031308.

$\checkmark 13$ Sato Y, Yamada T, Taniguchi R, Nagai K, Makiyama T, Okada H, Kataoka K, Ito H, Matsumori A, Sasayama S, Takatsu Y: Persistently increased serum concentrations of cardiac troponin $\mathrm{T}$ in patients with idiopathic dilated cardiomyopathy are predictive of adverse outcomes. Circulation 2001;103:369-374.

-14 Sato Y, Kataoka K, Matsumori A, Sasayama S, Yamada T, Ito H, Takatsu Y: Measuring serum aminoterminal type III procollagen peptide, 7S domain of type IV collagen, and cardiac troponin $\mathrm{T}$ in patients with idiopathic dilated cardiomyopathy and secondary cardiomyopathy. Heart 1997;78:505-508.

-15 Sato Y, Taniguchi R, Nagai K, Makiyama T, Okada H, Yamada T, Matsumori A, Takatsu $\mathrm{Y}$ : Measurements of cardiac troponin $\mathrm{T}$ in patients with hypertrophic cardiomyopathy. Heart 2003;89:659-960.
6 Soongswang J, Durongpisitkul K, Nana A, Laohaprasittiporn D, Kangkagate C, Punlee $\mathrm{K}$, Limpimwong $\mathrm{N}$ : Cardiac troponin $\mathrm{T}$ : a marker in the diagnosis of acute myocarditis in children. Pediatr Cardiol 2005;26:45-49.

17 Elkayam U, Tummala PP, Rao K, Akhter MW, Karaalp IS, Wani OR, Hameed A, Gviazda I, Shotan A: Maternal and fetal outcomes of subsequent pregnancies in women with peripartum cardiomyopathy. N Engl J Med 2001;344:1567-1571.

18 Lowbeer C, Stenvinkel P, Pecoits-Filho R, Heimburger O, Lindholm B, Gustafsson SA, Seeberger A: Elevated cardiac troponin $\mathrm{T}$ in predialysis patients is associated with inflammation and predicts mortality. J Intern Med 2003;253:153-160.

19 Seftel H, Susser M: Maternity and myocardial failure in African women. Br Heart J 1961;23:43-52.

20 O'Connell JB, Costanzo-Nordin MR, Subramanian R, Robinson JA, Wallis DE, Scanlon PJ, Gunnar RM: Peripartum cardiomyopathy: clinical, hemodynamic, histologic and prognostic characteristics. J Am Coll Cardiol 1986;8:52-56.

21 Sliwa K, Forster O, Libhaber E, Fett JD, Sundstrom JB, Hilfiker-Kleiner D, Ansari AA: Peripartum cardiomyopathy: inflammatory markers as predictors of outcome in 100 prospectively studied patients. Eur Heart J 2006;27:441-446.

22 Elkayam U, Akhter MW, Singh H, Khan S, Bitar F, Hameed A, Shotan A: Pregnancy-associated cardiomyopathy: clinical characteristics and a comparison between early and late presentation. Circulation 2005;111: 2050-2055.

23 Witlin AG, Mabie WC, Sibai BM: Peripartum cardiomyopathy: a longitudinal echocardiographic study. Am J Obstet Gynecol 1997;177:1129-1132.
24 Chapa JB, Heiberger HB, Weinert L, Decara J, Lang RM, Hibbard JU: Prognostic value of echocardiography in peripartum cardiomyopathy. Obstet Gynecol 2005;105:13031308.

25 Cole P, Cook F, Plappert T, Saltzman D, St John Sutton M: Longitudinal changes in left ventricular architecture and function in peripartum cardiomyopathy. Am J Cardiol 1987;60:871-876.

-26 Walsh JJ, Burch GE, Black WC, Ferrans VJ, Hibbs RG: Idiopathic myocardiopathy of the puerperium (postpartal heart disease). Circulation 1965;32:19-31.

27 McNeil AR, Marshall M, Ellis CJ, Hawkins $\mathrm{RC}$ : Why is troponin $\mathrm{T}$ increased in the serum of patients with end-stage renal disease? Clin Chem 1998;44:2377-2378.

28 Hibbard JU, Lindheimer M, Lang RM: A modified definition for peripartum cardiomyopathy and prognosis based on echocardiography. Obstet Gynecol 1999;94:311316.

29 Ardehali H, Kasper EK, Baughman KL: Peripartum cardiomyopathy. Minerva Cardioangiol 2003;51:41-48.

30 Mehta NJ, Mehta RN, Khan IA: Peripartum cardiomyopathy: clinical and therapeutic aspects. Angiology 2001;52:759-762.

-31 Perna ER, Macin SM, Canella JP, Augier N, Stival JL, Cialzeta JR, Pitzus AE, Garcia EH, Obregon R, Brizuela M, Barbagelata A: Ongoing myocardial injury in stable severe heart failure: value of cardiac troponin $\mathrm{T}$ monitoring for high-risk patient identification. Circulation 2004;110:2376-2382.

32 Sliwa K, Skudicky D, Bergemann A, Candy G, Puren A, Sareli P: Peripartum cardiomyopathy: analysis of clinical outcome, left ventricular function, plasma levels of cytokines and Fas/APO-1. J Am Coll Cardiol 2000;35: 701-705. 